\title{
Malignant and Nonmalignant Gene Signatures in Squamous Head and Neck Cancer
}

\author{
Maria J. Worsham, ${ }^{1}$ Mei Lu, ${ }^{2}$ Kang Mei Chen, ${ }^{1}$ Josena K. Stephen, ${ }^{1}$ \\ Shaleta Havard, ${ }^{1}$ and Vanessa P. Schweitzer ${ }^{1}$ \\ ${ }^{1}$ Department of Otolaryngology-Head \& Neck Surgery, Henry Ford Health System, Detroit, MI 48202, USA \\ ${ }^{2}$ Department of Biostatistics and Research Epidemiology, Henry Ford Health System, Detroit, MI 48202, USA
}

Correspondence should be addressed to Maria J. Worsham, mworsha1@hfhs.org

Received 7 December 2011; Revised 26 January 2012; Accepted 13 February 2012

Academic Editor: Mario A. Hermsen

Copyright (C) 2012 Maria J. Worsham et al. This is an open access article distributed under the Creative Commons Attribution License, which permits unrestricted use, distribution, and reproduction in any medium, provided the original work is properly cited.

\begin{abstract}
Genetic events specific to the pathogenesis of malignancy can offer clues to the tumorigenesis process. The objective of this study was to identify gene alterations that differentiate tumor and nontumor lesions in squamous head and neck cancer (HNSCC). DNA from 220 primary HNSCC with concurrently present tumor and nontumor lesions from the same patient was interrogated for genomic alterations of loss or gain of copy. Conditional logistic regression dealt with tumor and non-tumor records within a patient. Of 113 genes, 53 had univariate effects $(P<0.01)$, of which 16 genes remained in the multivariable model with $P<0.01$. The model had a C-index (ROC) of 0.93. Loss of CDKN2B and gain of BCL6, FGF3, and PTP4A3 predicted tumor. Loss of BAK1 and CCND1 and gain of $S T C H$ predicted nontumor. This highly powered model assigned alterations in 16 genes specific for malignant versus nonmalignant lesions, supporting their contribution to the pathogenesis of HNSCC as well as their potential utility as relevant targets for further evaluation as markers of early detection and progression.
\end{abstract}

\section{Introduction}

Knowledge of the genetic mechanisms that drive cancer growth and development is important in understanding the pathogenesis of malignancy and provides insights into the tumorigenesis process. The underlying hypothesis is that behavior of tumor cells is determined by genetic changes that alter cell growth, cell differentiation, programmed cell death, and cell migration. Cancer is the result of transformation from a normal to a malignant cell that results from accumulated mutations. Acquisition of a fully malignant phenotype in colon cancer is thought to occur because of multiple steps whose targets are alterations of growth-promoting oncogenes and growth-inhibiting cancer suppressor genes. The evolution in transformation from a normal squamous epithelial cell to a cancer cell is likewise assumed to require several steps, some defined by genetic alteration.

Genetic alterations provide means of identifying tumor cells as well as defining changes that presumably determine biological differences from their normal counterparts. Chromosome aberrations have served as landmarks to identify cancer genes in many tumor types; however, individual gene loci altered in tumors cannot be deduced solely from the type of chromosome rearrangement [1]. Historically, the molecular pathogenesis of cancer has been teased out one gene at a time. Recent high-throughput genomewide candidate strategies such as the multiplex ligation-dependent probe amplification (MLPA) assay [2] to identify specific genes for gain and loss concurred with chromosomal aberrations and provide a novel index to estimate the extent of genomic abnormality with disease progression [1].

Molecular genetic prognosticators can influence prevention, diagnosis, appropriateness of adjuvant chemotherapy, and, possibly, the chemotherapeutic regimen of cancer patients. Dissecting out processes specific to the pathogenesis of malignancy can distill key genetic biomarkers of HNSCC etiology, transformation, and progression.

In this study, in a primary HNSCC cohort of 220 patients, with both tumor and nontumor lesions within a biopsy (tumor and nontumor from the same patient), we examined gene alterations of loss and gain to derive multivariate 
predictive models to discriminate malignant from nonmalignant lesions.

\section{Methods}

2.1. Patient Cohort. Cohort subjects were male and female patients 18 years and older who underwent a HNSCC tissue biopsy at the Henry Ford Health System from 1986-2006. The use of formalin-fixed paraffin-embedded tissue blocks from patients with both tumor and nontumor records within the same biopsy and the collection of related patient information were approved by the Henry Ford Health System Institutional Review Board (IRB) Committee.

In the event a study subject had more than one eligible biopsy over the course of the study period, the primary biopsy was the index biopsy and the pathology report date marked the study enrollment (index) date. Cohort subjects missing biopsy tissue blocks or insufficient tissue for molecular analysis were excluded.

2.2. Histopathology. Pathology review of paraffin-embedded tissue sections captured all lesion types in a biopsy to include normal squamous epithelium, squamous dysplasia whether classified as mild, moderate, or severe/carcinoma in situ, and tumor. Severe dysplasia and carcinoma in situ lesions were grouped with tumor and considered as malignant outcomes.

\subsection{Molecular Analysis}

2.3.1. Processing Lesion Specimens for Molecular Analysis. DNA was obtained from either whole 5 micron tissue sections (if the tissue block contained predominantly tumor or nontumor tissue) or from microdissected tissue as previously described [3]. Briefly, concurrently present tumor (severe dysplasia, carcinoma in situ, tumor) and nontumor (normal, mild/moderate dysplasia) lesions in the same paraffin-embedded formalin fixed tissue block were marked by the pathologist and individual lesions were microdissected from 5 micron sections mounted on glass slides using a single-use disposable scalpel blade under a dissecting microscope. This procedure minimizes mixing of normal and tumor subpopulations and yields lesion and tumor samples estimated to be at least $90 \%$ free from contamination with normal cells $[3,4]$.

\subsubsection{The Multiplex Ligation-Dependent Probe Amplification} (MLPA) Assay. MLPA has several advantages. It establishes the copy number of up to 41 nucleic acid sequences in one single reaction. MLPA probes are able to discriminate between sequences that differ in only one nucleotide. Moreover, MLPA reactions require a minimum of only $20 \mathrm{ng}$ human DNA making it especially amenable for PCR of DNA from formalin-fixed paraffin-embedded head and neck tissues [3$5]$.

Starting with, approximately, 20-50 ng of genomic DNA, for each subject in the cohort, DNA from tumor and nontumor is amplified for 122 probes (113 unique genes associated with cancer including HNSCC) as separate sets of three reactions (probe sets p005, p006, p007, MRC Holland, Amsterdam). Briefly, DNA, diluted with water to a total volume of $5 \mu \mathrm{L}$, is denatured and fragmented by heating for $5 \mathrm{~min}-$ utes at $98^{\circ} \mathrm{C}$ in a thermocycler. Binary MLPA probes are added and allowed to hybridize to their targets during an $16 \mathrm{hr}$ incubation at $60^{\circ} \mathrm{C}$, followed by the addition of dilution buffer and a ligase enzyme (MRC Holland, Amsterdam). During the 15 -minute incubation at $60^{\circ} \mathrm{C}$, the two parts of a probe become ligated to each other and become an amplifiable molecule if the complementary sequence is present in the DNA sample. This is followed by the addition of PCR primers, dNTPs and Taq polymerase, followed by the following cycles: one at $1 \min 95^{\circ} \mathrm{C}, 10$ cycles: $30 \sec 95^{\circ} \mathrm{C}, 30 \sec 70^{\circ} \mathrm{C}$, $1 \mathrm{~min} 72^{\circ} \mathrm{C} ; 30$ cycles at $30 \sec 95^{\circ} \mathrm{C}, 30 \sec 60^{\circ} \mathrm{C}, 1 \mathrm{~min} 72^{\circ} \mathrm{C}$. The same primer pair, one of which is tagged with a fluorescent dye, amplifies all (ligated) probes. The relative amounts of PCR product obtained reflect the relative amounts of ligated probes at the start of the PCR reaction. Amplification products are analyzed on a DNA sequencer (Applied Biosystems, Foster City, Ca), quantified and interpreted as previously described $[1,3,4,6-8]$.

2.4. Statistical Analysis. Conditional logistic regression modeling was used to address tumor and nontumor lesions within the primary biopsy in an HNSCC patient. Analysis began by testing individual genes as risk predictors/discriminators for tumor and nontumor (univariate analysis). Genes with individual risks in a univariate analysis $(P<0.01)$ were candidates for the first multivariable model. Prior to multivariable modeling, genes were evaluated for their correlation and missing values. Highly correlated genes (correlation coefficient $[r]>0.7)$ or genes with larger missing values $(>5 \%)$ were fitted separately along with other uncorrelated $(r<0.7)$ genes. The stepwise model selection was considered. The final model included genes with $P<0.01$ along with odds ratios for loss or gain as risk predictors. The $\mathrm{C}$-index/ROC (the receiver operating characteristic $\{$ ROC $\}$ curve), in a range of 0 to 1 , is a measure of the model's predictive ability, where 0.5 indicates no discrimination and 0.7 or greater indicates that the model is predictive.

\section{Results and Discussion}

3.1. Results. Matched tumor and nontumor lesions within each patient in the 220 primary HNSCC cohort comprised a total of 1076 tissue records. There were 504 normal/mild/ moderate dysplasia lesions (495: normal squamous epithelium, 6: mild dysplasia, 3: moderate dysplasia), and 572 tumor lesions (568: tumor, 1: severe dysplasia, 3: carcinoma in situ (CIS)). Squamous mucosal dysplasia whether classified as mild, moderate, or severe/carcinoma in situ is considered intraepithelial neoplasia, and, as a precursor lesion group is separate from normal and tumor. The number of precursor, lesions is very small (13) in comparison to normal epithelium (495) and carcinomas (568). Of the 572 tumor lesions, 193 (34\%) were laryngeal, $170(30 \%)$ were oral cavity, 151 (26\%) were pharyngeal (oropharyngeal/hypopharyngeal), 
TABLE 1: Genes alterations that predict malignant ( $M$, bolded) and nonmalignant (NM, italicized).

\begin{tabular}{|c|c|c|c|c|}
\hline Effect & Chromosome & Odds ratio estimate & Lower $\mathrm{CL}^{*}$ & Upper CL \\
\hline$\${ }^{\mathrm{M}} \mathrm{CTNNB1}$ loss versus normal & $3 \mathrm{p} 22$ & 2.682 & 1.394 & 5.162 \\
\hline${ }^{\mathrm{NM}} C T N N B 1$ gain versus normal & & 0.323 & 0.147 & 0.71 \\
\hline$B C L 6$ loss versus normal & $3 \mathrm{q} 27$ & 0.55 & 0.27 & 1.12 \\
\hline${ }^{\mathrm{M}} B C L 6$ gain versus normal & & 8.989 & 3.155 & 25.612 \\
\hline${ }^{\S \mathrm{M}} I L 2$ loss versus normal & $4 \mathrm{q} 26$ & 3.697 & 1.774 & 7.705 \\
\hline${ }^{\mathrm{NM}}$ IL2 gain versus normal & & 0.149 & 0.055 & 0.407 \\
\hline${ }^{\mathrm{NM}} B A K 1$ loss versus normal & $6 \mathrm{p} 21.3$ & 0.262 & 0.103 & 0.666 \\
\hline$B A K 1$ gain versus normal & & 0.438 & 0.192 & 0.999 \\
\hline${ }^{\S \mathrm{M}} L T A$ loss versus normal & $6 \mathrm{p} 21.3$ & 2.156 & 1.172 & 3.965 \\
\hline${ }^{\mathrm{NM}} L T A$ gain versus normal & & 0.266 & 0.108 & 0.655 \\
\hline$\S \mathrm{NM} F G F R 1$ loss versus normal & $8 \mathrm{p} 21$ & 0.275 & 0.126 & 0.598 \\
\hline${ }^{\mathrm{M}} F G F R 1$ gain versus normal & & 5.555 & 1.689 & 18.267 \\
\hline${ }^{\mathrm{NM}}$ PRKDC loss versus normal & $8 \mathrm{q} 11$ & 0.276 & 0.11 & 0.692 \\
\hline${ }^{\mathrm{M}} P R K D C$ gain versus normal & & 5.449 & 2.09 & 14.206 \\
\hline${ }_{\S \mathrm{NM}} M Y C$ loss versus normal & $8 \mathrm{q} 24.12$ & 0.221 & 0.097 & 0.503 \\
\hline${ }^{\mathrm{M}} M Y C$ gain versus normal & & 2.218 & 1.136 & 4.332 \\
\hline PTP4A3 loss versus normal & $8 \mathrm{q} 24.3$ & 0.493 & 0.214 & 1.135 \\
\hline${ }^{\mathrm{M}} P T P 4 A 3$ gain versus normal & & 12.158 & 3.461 & 42.71 \\
\hline${ }^{\S M} C D K N 2 A$ loss versus normal & $9 \mathrm{p} 21$ & 1.845 & 1.013 & 3.362 \\
\hline${ }^{\mathrm{NM}} C D K N 2 A$ gain versus normal & & 0.14 & 0.056 & 0.35 \\
\hline${ }^{\mathrm{M}} C D K N 2 B$ loss versus normal & $9 \mathrm{p} 21$ & 3.256 & 1.676 & 6.325 \\
\hline$C D K N 2 B$ gain versus normal & & 1.168 & 0.442 & 3.087 \\
\hline${ }^{\mathrm{M}} \mathrm{LMO} 2$ loss versus normal & $11 \mathrm{p} 13$ & 4.977 & 2.16 & 11.466 \\
\hline LMO2 gain versus normal & & 0.573 & 0.205 & 1.607 \\
\hline FGF3 loss versus normal & $11 \mathrm{q} 13$ & 0.882 & 0.447 & 1.741 \\
\hline${ }^{\mathrm{M}} F G F 3$ gain versus normal & & 7.819 & 3.286 & 18.604 \\
\hline${ }^{\mathrm{NM}} C C N D 1$ loss versus normal & $11 \mathrm{q} 13$ & 0.403 & 0.22 & 0.736 \\
\hline$C C N D 1$ gain versus normal & & 1.239 & 0.634 & 2.421 \\
\hline STCH loss versus normal & 21q11.1 & 1.788 & 0.833 & 3.839 \\
\hline${ }^{\mathrm{NM}} S T C H$ gain versus normal & & 0.124 & 0.043 & 0.359 \\
\hline${ }_{\S M} T F F 1$ loss versus normal & $21 \mathrm{q} 22.3$ & 3.019 & 1.514 & 6.02 \\
\hline${ }^{\mathrm{NM}} T F F 1$ gain versus normal & & 0.08 & 0.024 & 0.268 \\
\hline
\end{tabular}

${ }^{*}$ CL: confidence limit, ${ }^{\S}$ : genes with loss and gain signifying aneuploidy.

and $58(10 \%)$ were lesions in the other category (nasopharynx, nasal cavity, paranasal sinuses).

The 1076 lesions were distributed among 932 tissue blocks, of which $434(47 \%)$ had tumor lesions only, 363 (39\%) had nontumor lesions only, and 135 (14\%) tissue blocks had both tumor and nontumor lesions. Within a patient, the number of nontumor lesions ranged from 1 to 6 and from 1 to 7 for tumor lesions. Of the 220 patients with both tumor and nontumor within the same patient, 35 $(16 \%)$ had 1 tumor and nontumor record, $28(13 \%)$ had 2 tumor and nontumor records, 27 (12\%) had 3 tumor and nontumor records.

Fifty percent of cohort (110/220) subjects were Caucasian American (CA), 38\% (84/220) were self-reported as African American (AA), 4\% (9/220) were non-CA/non-AA (Hispanic: 2, Asian Pacific Islander: 2, Middle Eastern: 3, other: $2)$, and, in $8 \%(17 / 220)$, race was missing. Of the 220 patients, 25\% (55/220) were female and 75\% (165/220) were male.

The missing value for each gene was in a range of $0 \%$ to $4.2 \%$. Of the 113 unique genes (selected based on their association with cancer including HNSCC), 53 genes had univariate effects $(P<0.01)$ and were considered as the candidate genes for multivariable analyses. After the stepwise model selection, 16 genes remained in the multivariable model $(P<0.01)$ (Table 1$)$. The model had a C-index (receiver operating characteristic (ROC)) of 0.93 .

The 16 genes in the final model with alterations of loss and/or gain accounted for loci along 7 chromosomes: 3,4 , $6,8,9,11$, and 21 (Table 1 ). Of these, $50 \%$ were altered in both tumor and nontumor, with loss or gain reflective of chromosomal aneuploidy. This copy number instability favored loss of CDKN2A (9p21), CTNNB1 (3p21), IL2 (4q26), LTA (6p21.3), and TFF1 (21q22.3) in tumor, with 
corresponding gain in nontumor lesions, and gain of FGFR1 (8p21), MYC (8q24.12), and PRKDC (8q11) in tumor, with corresponding loss in nontumor (Table 1$)$.

Loss of CDKN2B (9p21) and LMO2 (11p13) and gain of BCL6 (3q27), FGF3 (11q13), and PTP4A3 (8q24.3) predicted tumor. Loss of $B A K 1$ (6p21.3) and CCND1 (11q13) and gain of STCH (21q11.1) predicted nontumor (Table 1).

Analysis excluding the 6 mild dysplasia and 3 moderate dysplasia lesions from the nontumor group and the 1 severe dysplasia lesion and 3 CIS from the tumor group generated an identical multivariable model outcome.

3.2. Discussion. Cancerous tissue in most cases has a distinctive appearance under the microscope. Distinguishing traits include a large number of dividing cells, variation in nuclear size and shape, variation in cell size and shape, loss of specialized cell features, loss of normal tissue organization, and a poorly defined tumor boundary. Biopsy and microscopical examination can also distinguish malignancy, precursor lesions of carcinoma in situ, mild, moderate, and severe dysplasia, and less reliably, hyperplasia, from normal appearing tissue.

In the multistep process of tumorigenesis, hyperplasia, which refers to tissue growth based on an excessive rate of cell division, leading to a larger than usual number of cells but with a normal orderly arrangement of cells within the tissue, and considered reversible, is thought to precede dysplasia. Dysplasia, an abnormal type of excessive cell proliferation characterized by loss of normal tissue arrangement and cell structure, may revert to normal behavior, but, occasionally, these lesions gradually become malignant. Distinguishing true precursor lesions on the basis of morphology alone is often unreliable. In the molecular progression of HNSCC, normal or minimal dysplasia often harbors abnormal genotypes [9], which do not necessarily correlate with observable changes in phenotype (morphology) $[10,11]$.

In this study, 16 gene alterations with significant discriminatory ability differentiated malignant HNSCC from nonmalignant tissue. For matched tumor and nontumor lesions from the same patient within the 220 primary HNSCC study cohort, examined for alterations in 113 unique genes with association to head and neck cancer, molecular alterations in "normal" appearing epithelium within the environment of a malignant biopsy harbored genotypic abnormalities that set them apart from malignant tissue.

The 16 genes in the final model span loci along 7 chromosomes: 3p21: CTNNB1, 3q27: BCL6; 4q26: IL2; 6p21.3: BAK1 and LTA; 8p12: FGFR, 8q24.12: MYC, 8q24.3: PTP4A3; 9p21: CDKN2A, CDKN2B; 11p13: LMO2, 11q13: CCND1, FGF3; 21q11.1: STCH, 21q22.3: TFF1. Gene alterations at these loci restate reported cytogenetic [7, 12-22] and molecularly altered regions by $\mathrm{LOH}$ and array $\mathrm{CGH}$ studies in HNSCC $[1,5,7,23-28]$. Additionally, copy number loss in tumor and corresponding gain in nontumor, and vice versa, advocate aneuploidy events. The latter are highlighted for loss of CDKN2A (9p21), CTNNB1 (3p22), IL2 (4q26), LTA (6p21.3), and TFF1 (21q22.3) in tumor, with corresponding gain in nontumor lesions, and gain of FGFR1 (8p12), MYC (8q24.12), and PRKDC (8q11) in tumor, with corresponding loss in nontumor. Chromosomal instability occurs early along the tumorigenesis continuum and aneuploidy at the 9 p21 locus affecting corresponding loss and gain in tumor and normal tissue, respectively, concurs with the proposed postulated model of molecular carcinogenesis for HNSCC [29].

In HNSCC, chromosomal aberrations on the long arm of chromosome 3, resulting in gain of distal 3q segments, have been reported as recurring karyotypic alterations [22]. Gain of $3 q$ is supported by increased copy number (3-4 copies) of PIK3CA at 3q26.3, MME (3q25.1), and BCL6 genes at 3q27 [1] in HNSCC. In this study, gain of BCL6 was significantly associated with tumor lesions. The protein encoded by BCL6 is a zinc finger transcription factor and acts as a sequencespecific repressor of transcription.

Another chromosome 3 gene, CTNNB1 (catenin beta-1) in the short arm at $3 \mathrm{p} 21$, is an adherens junction protein, closely associated with adhesion, invasion, and metastasis in different types of tumors, including SCC of the tongue [30]. The 3 p21 region had the highest rate of allelic deletion $(63 \%)$ in HNSCC [31] and is supported by loss of CTNNB1 in tumor lesions in this study. Corresponding gain of CTNNB1 copy number in nontumor lesions underscores chromosomal instability and ensuing aneuploidy as early events in the tumorigenesis process.

Loss and corresponding gain of IL2 at 4q26 was significantly associated with tumor and nontumor lesions, respectively. The IL2 protein is produced by T cells in response to antigenic or mitogenic stimulation and is required for T-cell proliferation and other activities crucial to regulation of the immune response.

$B A K 1(6 \mathrm{p} 21.3)$ is a proapoptotic member of the $B C L-2$ family of genes that are involved in regulation of programmed cell death, and its increased expression had poorer disease-specific survival in oral tongue squamous cell carcinomas [32]. As a corollary to increased expression [32], in this study, loss of $B A K 1$ was a nontumor-specific event.

Gene alterations were noted for 4 genes on chromosome 8 , three on $8 \mathrm{q}$ and one on $8 \mathrm{p}$. Gains or amplifications involving chromosome arm $8 \mathrm{q}$ are one of the most recurrent chromosomal alterations in head and neck tumors. The human protein tyrosine phosphatase type IVA, member 3, also known as PTP4A3, is located at 8q24.3 [33]. The protein encoded by this gene is a cell signaling molecule that participates in every aspect of cellular physiologic and pathologic processes [33]. Recent studies [34, 35] suggest that an excess PTP4A3 may play a key role in the acquisition of metastatic potential of tumor cells. This study further supports gain of PTP4A3 as a malignancy-associated alteration [36] in HNSCC.

The MYC oncogene, located at 8q24.12, encodes a transcription factor that plays a key role in cell proliferation, differentiation, and apoptosis [37]. Gain of MYC was significant for laryngeal tumor progression [38], and the concomitant over expression of MYC and $p 53$ oncogenes had worse disease-free survival suggesting a role for $p 53$ and MYC genes in progression of HNSCC [39]. In this study, gain of $M Y C$ significantly discriminated tumor from nontumor tissue. 
The corresponding loss of MYC copy number in nontumor suggests aneuploidy as a likely destabilizing event.

DNA double-strand breaks repair pathway has been implicated in maintaining genomic integrity via suppression of chromosomal rearrangements. PRKDC (protein kinase, DNA-activated, catalytic polypeptide) is associated with chromosomal instability with risk of breast and uterine cervix cancer [40]. In this study, genomic instability at the 8q11 locus favored gain of PRKDC copy number in tumor and corresponding loss in nontumor.

FGFR-1, located at 8p21, had gain of copy number in tumor and corresponding loss in nontumor. FGFR-1 expression has been detected in thyroid carcinoma [41] and in oral squamous cell carcinomas (OSCC). Amplification of FGFR1 detected by FISH analysis on OSCC tissue microarray sections contributed to oral carcinogenesis at an early stage of development [42].

Genetic alterations at the 9p21 locus have been linked to malignant progression in HNSCC [43, 44]. CDKN2A and $C D K N 2 B$ genes map to $9 \mathrm{p} 21$ and are in tandem, spanning a region of approximately $80 \mathrm{~kb}[45,46]$. Inactivation of the CDKN2A (p14), CDKN2B (p15), and CDKN2A (p16) genes is a frequent event in human oral squamous cell carcinomas [47]. The main modes of $p 16^{\mathrm{INK} 4 \mathrm{a}}$ inactivation in HNSCC are known to include homozygous deletions (40-60\%), mutations (15-20\%), and gene hypermethylation events (5\%) [47-49]. This study supports loss of CDKN2A and CDKN2B genes as independent predictors of tumor in HNSCC patients.

Amplification of the 11q13 amplicon is driven by multiple genes, rather than only one or two genes at this site [5052]. In this study, four genes at the 11q13 locus were interrogated, CCND1, FGF3, EMS1, and RELA, of which, gain of $C C N D 1, F G F 3$, and EMS1 were univariate $(P<0.0001)$ predictors of tumor. Multivariate analysis $(P<0.01)$ retained gain of FGF3 in tumor and loss in nontumor, supporting involvement of amplification/gain of copy number of this gene in HNSCC [53, 54]. FGF3 belongs to the basic fibroblast growth factor (FGF) gene family with a role in several important cellular processes, including regulation of cell growth and division, determination of cell type, formation of blood vessels, wound healing, and embryo development. In HNSCC, FGF3 had a significantly higher frequency of amplification in hypopharyngeal tumors [55]. Loss of CCND1 was significantly associated with nontumor lesions in this study cohort, suggesting genomic instability/chromosomal aneuploidy events in the direction of corresponding gain of CCND1 in tumor lesions $(P<0.001$, univariate analysis). Overexpression and/or amplification of CCND1 is reported in 35\%-65\% of patients with HNSCC and is associated with poor prognosis [56-58]. Its expression is deregulated in preinvasive lesions adjacent to invasive tumors and is associated with increased chromosomal instability and the likelihood of subsequent gene amplification. [59,60] Loss of CCND1 copy number in nontumor tissues in this study may reflect very early genomic instability at this chromosomal locus and supports reports of CCND1 deregulation in preinvasive lesions of the upper aerodigestive tract with associated increased risk for the development of cancer accompanied by histologic progression during and after chemopreventive intervention $[61,62]$. Though corresponding gain of CCND1 was not retained in the final multivariate model, $24 \%$ of tumor lesions had copy number gain. Loss of LMO2 (also known as RBTN2 and TTG2), located at $11 \mathrm{p} 13$, predicted tumor lesions. This gene encodes a transcriptional cofactor critical for the development of hematopoietic stem cells [63].

Gain of copy number at two 21q loci, TFF1 (21q22.3) and STCH (21q11.1), predicted nontumor lesions, and corresponding loss of TFF1 was a significantly associated with tumor lesions. Loss of TFF1 in tumor lesions with corresponding gain in nontumor supports genomic instability as a concerted early tumorigenesis event. STCH (stress 70 protein chaperone), at 21q11.1, a member of the heat shock protein 70 (HSP70) superfamily with cell-protective functions, was previously identified as a candidate gene for susceptibility to stomach cancer by genetic analyses [64]. STCH copy number gain in nontumor lesions remained in the final model as an independent predictor of nonmalignant tissue (corresponding loss in tumor lesions remained a univariate variable, $P<0.001)$.

The model's discriminatory abilities (C-index/ROC of 0.93 ) support molecular distinctiveness of malignant versus nonmalignant tissue with significant predictive power. The latter is of particular significance because normal samples from patients with head and neck cancer, especially in the neighborhood of the tumor, can be genetically altered (field cancerization). The proximity of tumor and nontumor lesions, therefore, makes it harder to discriminate between these two entities. However, the relatively large number of tissue records $(n=1,076)$ from 220 patients was a factor in overcoming these challenges to yield a robust model with excellent ability $(\mathrm{C}$-index $=0.93$ ) to discriminate malignant and nonmalignant tissue within the same patient.

Genetic alterations at 16 chromosomal loci underscore the association of already known genes as well as newer gene targets in HNSCC pathogenesis. The sixteen gene predictors spanning loci along 7 chromosomes cover an array of essential functions that ensure normal homeostasis to include DNA repair (PRKDC), initiation of carcinogenesis (TFF1), immune surveillance (IL2, LTA), cell cycle regulators (CDKN2A, CDKN2B), apoptosis (BAK1, STCH), regulation of cell proliferation and differentiation (CCND1, FGF3, $M Y C$ ), transcription factors (BCL6), stem cell hematopoiesis (LMO2), adhesion, invasion and metastasis (CTNNB1, FGFR1), and acquisition of metastatic potential of tumor cells (PTP4A3), implicating these genes as key players in the tumorigenesis continuum.

\section{Conclusion}

Genomic instability, a hallmark of malignant transformation, promotes a wide range of mutations, including chromosome deletions, gene amplifications, translocations, and polyploidy [40]. In this study, the directional loss and gain for several genes underscored the contribution of aneuploidy in early HNSCC tumorigenesis. Our data support distinct 
genetic signatures that discriminate malignant and nonmalignant tissue in HNSCC. The 16 gene alteration signature in this study suggests finely choreographed genomic instability events to achieve biological distinctiveness, providing clues to the drivers in invasive cancers as well as insight into gene rearrangements that might arise in nonmalignant lesions. The gene sets meet statistical rigor to segregate malignant squamous carcinoma lesions from nonmalignant lesions, providing an opportunity for researchers to investigate these cancer-associated genes as potential targets of therapy either as single targets or as sets of targets when these occur in the same cancer lesion.

\section{Acknowledgment}

This research was supported by NIH R01 DE 15990 (M. J. Worsham).

\section{References}

[1] M. J. Worsham, G. Pals, J. P. Schouten et al., "Delineating genetic pathways of disease progression in head and neck squamous cell carcinoma," Archives of Otolaryngology-Head and Neck Surgery, vol. 129, no. 7, pp. 702-708, 2003.

[2] J. P. Schouten, C. J. McElgunn, R. Waaijer, D. Zwijnenburg, F. Diepvens, and G. Pals, "Relative quantification of $40 \mathrm{nu}$ cleic acid sequences by multiplex ligation-dependent probe amplification," Nucleic Acids Research, vol. 30, no. 12, p. e57, 2002.

[3] U. Raju, M. Lu, S. Sethi, H. Qureshi, S. R. Wolman, and M. J. Worsham, "Molecular classification of breast carcinoma in situ," Current Genomics, vol. 7, no. 8, pp. 523-532, 2006.

[4] J. K. Stephen, L. E. Vaught, M. C. Kang et al., "An epigenetically derived monoclonal origin for recurrent respiratory papillomatosis," Archives of Otolaryngology-Head and Neck Surgery, vol. 133, no. 7, pp. 684-692, 2007.

[5] O. Saglam, V. Shah, and M. J. Worsham, "Molecular differentiation of early and late stage laryngeal squamous cell carcinoma: an exploratory analysis," Diagnostic Molecular Pathology, vol. 16, no. 4, pp. 218-221, 2007.

[6] M. J. Worsham, G. Pals, J. P. Schouten et al., "High-resolution mapping of molecular events associated with immortalization, transformation, and progression to breast cancer in the MCF10 model," Breast Cancer Research and Treatment, vol. 96, no. 2, pp. 177-186, 2006.

[7] M. J. Worsham, M. C. Kang, N. Tiwari et al., "Fine-mapping loss of gene architecture at the CDKN2B (p15INK4b), CDKN2A (p14ARF, p16INK4a), and MTAP genes in head and neck squamous cell carcinoma," Archives of OtolaryngologyHead and Neck Surgery, vol. 132, no. 4, pp. 409-415, 2006.

[8] J. P. Kunjoonju, M. Raitanen, S. Grénman, N. Tiwari, and M. J. Worsham, "Identification of individual genes altered in squamous cell carcinoma of the vulva," Genes Chromosomes and Cancer, vol. 44, no. 2, pp. 185-193, 2005.

[9] A. Forastiere, W. Koch, A. Trotti, and D. Sidransky, "Head and neck cancer," New England Journal of Medicine, vol. 345, no. 26, pp. 1890-1900, 2001.

[10] M. P. Rosin, X. Cheng, C. Poh et al., "Use of allelic loss to predict malignant risk for low-grade oral epithelial dysplasia," Clinical Cancer Research, vol. 6, no. 2, pp. 357-362, 2000.

[11] M. Partridge, S. Pateromichelakis, E. Phillips, G. G. Emilion, R. P. A'Hern, and J. D. Langdon, "A case-control study confirms that microsatellite assay can identify patients at risk of developing oral squamous cell carcinoma within a field of cancerization," Cancer Research, vol. 60, no. 14, pp. 3893-3898, 2000.

[12] T. E. Carey, C. J. Frank, J. R. Raval et al., "Identifying genetic changes associated with tumor progression in squamous cell carcinoma," Acta Oto-Laryngologica, no. 529, pp. 229-232, 1997.

[13] D. L. Buchhagen, M. J. Worsham, D. L. Van Dyke, and T. E. Carey, "Two regions of homozygosity on chromosome $3 \mathrm{p}$ in squamous cell carcinoma of the head and neck: comparison with cytogenetic analysis," Head and Neck, vol. 18, no. 6, pp. 529-537, 1996.

[14] T. E. Carey, M. J. Worsham, and D. L. Van Dyke, "Chromosomal biomarkers in the clonal evolution of head and neck squamous neoplasia," Journal of Cellular Biochemistry, vol. 52, pp. 213-222, 1993.

[15] T. E. Carey, D. L. van Dyke, and M. J. Worsham, "Nonrandom chromosome aberrations and clonal populations in head and neck cancer," Anticancer Research, vol. 13, no. 6 B, pp. 25612567, 1993.

[16] C. R. Bradford, K. A. Kimmel, D. L. Van Dyke et al., “11p deletions and breakpoints in squamous cell carcinoma: association with altered reactivity with the UM-E7 antibody," Genes Chromosomes and Cancer, vol. 3, no. 4, pp. 272-282, 1991.

[17] T. E. Carey, D. L. Van Dyke, M. J. Worsham et al., "Characterization of human laryngeal primary and metastatic squamous cell carcinoma cell lines UM-SCC-17A and UM-SCC-17B," Cancer Research, vol. 49, no. 21, pp. 6098-6107, 1989.

[18] M. J. Worsham, M. J. Benninger, R. J. Zarbo, T. E. Carey, and D. L. Van Dyke, "Deletion 9p22-pter and loss of Y as primary chromosome abnormalities in a squamous cell carcinoma of the vocal cord," Genes Chromosomes and Cancer, vol. 6, no. 1, pp. 58-60, 1993.

[19] M. J. Worsham, T. E. Carey, M. S. Benninger et al., "Clonal cytogenetic evolution in a squamous cell carcinoma of the skin from a xeroderma pigmentosum patient," Genes Chromosomes and Cancer, vol. 7, no. 3, pp. 158-164, 1993.

[20] M. J. Worsham, S. R. Wolman, T. E. Carey, R. J. Zarbo, M. S. Benninger, and D. L. Van Dyke, "Common clonal origin of synchronous primary head and neck squamous cell carcinomas: analysis by tumor karyotypes and fluorescence in situ hybridization," Human Pathology, vol. 26, no. 3, pp. 251-261, 1995.

[21] M. J. Worsham, S. R. Wolman, T. E. Carey, R. J. Zarbo, M. S. Benninger, and D. L. Van Dyke, "Chromosomal aberrations identified in culture of squamous carcinomas are confirmed by fluorescence in situ hybridisation," Journal of Clinical Pathology, vol. 52, no. 1, pp. 42-46, 1999.

[22] D. L. van Dyke, M. J. Worsham, M. S. Benninger et al., "Recurrent cytogenetic abnormalities in squamous cell carcinomas of the head and neck region," Genes Chromosomes and Cancer, vol. 9, no. 3, pp. 192-206, 1994.

[23] H. de Schutter, M. Spaepen, W. H. Mc Bride, and S. Nuyts, "The clinical relevance of microsatellite alterations in head and neck squamous cell carcinoma: a critical review," European Journal of Human Genetics, vol. 15, no. 7, pp. 734-741, 2007.

[24] X. Zhou, R. C. K. Jordan, Y. Li, B. L. Huang, and D. T. W. Wong, "Frequent allelic imbalances at $8 p$ and $11 q 22$ in oral and oropharyngeal epithelial dysplastic lesions," Cancer Genetics and Cytogenetics, vol. 161, no. 1, pp. 86-89, 2005.

[25] S. W. Coon, A. T. Savera, R. J. Zarbo et al., "Prognostic implications of loss of heterozygosity at 8 p21 and 9p21 in head and 
neck squamous cell carcinoma," International Journal of Cancer, vol. 111, no. 2, pp. 206-212, 2004.

[26] J. Akervall, "Genomic screening of head and neck cancer and its implications for therapy planning," European Archives of Oto-Rhino-Laryngology, vol. 263, no. 4, pp. 297-304, 2006.

[27] Q. Huang, G. P. Yu, S. A. McCormick et al., "Genetic differences detected by comparative genomic hybridization in head and neck squamous cell carcinomas from different tumor sites: construction of oncogenetic trees for tumor progression," Genes Chromosomes and Cancer, vol. 34, no. 2, pp. 224-233, 2002.

[28] K. Gotte, S. C. Tremmel, S. Popp et al., "Intratumoral genomic heterogeneity in advanced head and neck cancer detected by comparative genomic hybridization," Advances in Oto-RhinoLaryngology, vol. 62, pp. 38-48, 2005.

[29] C. R. Leemans, B. J. M. Braakhuis, and R. H. Brakenhoff, "The molecular biology of head and neck cancer," Nature Reviews Cancer, vol. 11, no. 1, pp. 9-22, 2011.

[30] S. Li, J. Jiao, Z. Lu, and M. Zhang, "An essential role for Ncadherin and $\beta$-catenin for progression in tongue squamous cell carcinoma and their effect on invasion and metastasis of Tca8113 tongue cancer cells," Oncology Reports, vol. 21, no. 5, pp. 1223-1233, 2009.

[31] M. V. Gonzalez, M. F. Pello, P. Ablanedo, C. Suárez, V. Alvarez, and E. Coto, "Chromosome $3 p$ loss of heterozygosity and mutation analysis of the FHIT and $\beta$-cat genes in squamous cell carcinoma of the head and neck," Journal of Clinical Pathology, vol. 51, no. 7, pp. 520-524, 1998 .

[32] X. Xie, O. P. Clausen, and M. Boysen, "Prognostic value of Bak expression in oral tongue squamous cell carcinomas," Oncology Reports, vol. 10, no. 2, pp. 369-374, 2003.

[33] Q. Zeng, W. Hong, and Y. H. Tan, "Mouse PRL-2 and PRL-3, two potentially prenylated protein tyrosine phosphatases homologous to PRL-1," Biochemical and Biophysical Research Communications, vol. 244, no. 2, pp. 421-427, 1998.

[34] F. Polato, A. Codegoni, R. Fruscio et al., "PRL-3 phosphatase is implicated in ovarian cancer growth," Clinical Cancer Research, vol. 11, no. 19, pp. 6835-6839, 2005.

[35] U. A. Miskad, S. Semba, H. Kato, and H. Yokozaki, "Expression of PRL-3 phosphatase in human gastric carcinomas: close correlation with invasion and metastasis," Pathobiology, vol. 71, no. 4, pp. 176-184, 2004.

[36] M. Canel, P. Secades, M. Garzón-Arango et al., "Involvement of focal adhesion kinase in cellular invasion of head and neck squamous cell carcinomas via regulation of MMP-2 expression," British Journal of Cancer, vol. 98, no. 7, pp. 1274-1284, 2008.

[37] C. V. Dang, "c-Myc target genes involved in cell growth, apoptosis, and metabolism," Molecular and Cellular Biology, vol. 19, no. 1, pp. 1-11, 1999.

[38] D. K. Koynova, V. S. Tsenova, and D. I. Toncheva, "Tissue microarray analysis of C-MYC oncogene copy number changes in larynx carcinoma," ORL, vol. 67, no. 2, pp. 92-95, 2005.

[39] A. F. L. Waitzberg, S. Nonogaki, I. N. Nishimoto et al., "Clinical significance of c-myc and p53 expression in head and neck squamous cell carcinomas," Cancer Detection and Prevention, vol. 28, no. 3, pp. 178-186, 2004.

[40] M. Someya, K. I. Sakata, Y. Matsumoto et al., "The association of DNA-dependent protein kinase activity with chromosomal instability and risk of cancer," Carcinogenesis, vol. 27, no. 1, pp. 117-122, 2006.

[41] R. S. Bernard, L. Zheng, W. Liu, D. Winer, S. L. Asa, and S. Ezzat, "Fibroblast growth factor receptors as molecular targets in thyroid carcinoma," Endocrinology, vol. 146, no. 3, pp. 1145-1153, 2005.

[42] K. Freier, C. Schwaenen, C. Sticht et al., "Recurrent FGFR1 amplification and high FGFR1 protein expression in oral squamous cell carcinoma (OSCC)," Oral Oncology, vol. 43, no. 1, pp. 60-66, 2007.

[43] W. M. Lydiatt, B. J. Davidson, S. P. Schantz, S. Caruana, and R. S. K. Chaganti, "9p21 deletion correlates with recurrence in head and neck cancer," Head and Neck, vol. 20, no. 2, pp. 113-118, 1998.

[44] A. L. Reed, J. Califano, P. Cairns et al., "High frequency of p16 (CDKN2/MTS-1/INK4A) inactivation in head and neck squamous cell carcinoma," Cancer Research, vol. 56, no. 16, pp. 3630-3633, 1996.

[45] C. J. Sherr, "Cancer cell cycles," Science, vol. 274, no. 5293, pp. 1672-1674, 1996.

[46] C. J. Sherr, "The pezcoller lecture: cancer cell cycles revisited," Cancer Research, vol. 60, no. 14, pp. 3689-3695, 2000.

[47] S. Shintani, Y. Nakahara, M. Mihara, Y. Ueyama, and T. Matsumura, "Inactivation of the p14ARF, p15INK4B and p16INK4A genes is a frequent event in human oral squamous cell carcinomas," Oral Oncology, vol. 37, no. 6, pp. 498-504, 2001.

[48] E. P. Xing, Y. Nie, Y. Song et al., "Mechanisms of inactivation of p14(ARF), p15(INK4b), and p16(INK4a) genes in human esophageal squamous cell carcinoma," Clinical Cancer Research, vol. 5, no. 10, pp. 2704-2713, 1999.

[49] J. W. Rocco and D. Sidransky, "p16(MTS-1/CDKN2/INK4a) in cancer progression," Experimental Cell Research, vol. 264, no. 1, pp. 42-55, 2001.

[50] P. Gaudray, P. Szepetowski, C. Escot, D. Birnbaum, and C. Theillet, "DNA amplification at 11q13 in human cancer: from complexity to perplexity," Mutation Research, vol. 276, no. 3, pp. 317-328, 1992.

[51] E. Schuuring, "The involvement of the chromosome 11q13 region in human malignancies: cyclin D1 and EMS1 are two new candidate oncogenes-a review," Gene, vol. 159, no. 1, pp. 8396, 1995.

[52] X. Huang, S. M. Gollin, S. Raja, and T. E. Godfrey, "Highresolution mapping of the 11q13 amplicon and identification of a gene, TAOS1, that is amplified and overexpressed in oral cancer cells," Proceedings of the National Academy of Sciences of the United States of America, vol. 99, no. 17, pp. 11369-11374, 2002.

[53] K. D. Somers, S. L. Cartwright, and G. L. Schechter, "Amplification of the int-2 gene in human head and neck squamous cell carcinomas," Oncogene, vol. 5, no. 6, pp. 915-920, 1990.

[54] J. S. Rubin, L. Qiu, and P. Etkind, "Amplification of the Int-2 gene in head and neck squamous cell carcinoma," Journal of Laryngology and Otology, vol. 109, no. 1, pp. 72-76, 1995.

[55] J. P. Rodrigo, C. Suárez, M. V. González et al., "Variability of genetic alterations in different sites of head and neck cancer," Laryngoscope, vol. 111, no. 7, pp. 1297-1301, 2001.

[56] J. A. Akervall, R. J. A. M. Michalides, H. Mineta et al., "Amplification of cyclin D1 in squamous cell carcinoma of the head and neck and the prognostic value of chromosomal abnormalities and cyclin D1 overexpression," Cancer, vol. 79, no. 2, pp. 380-389, 1997.

[57] R. J. A. M. Michalides, N. M. J. Van Veelen, P. M. P. Kristel et al., "Overexpression of cyclin D1 indicates a poor prognosis in squamous cell carcinoma of the head and neck," Archives of Otolaryngology-Head and Neck Surgery, vol. 123, no. 5, pp. 497-502, 1997. 
[58] J. A. Akervall, Y. Jin, J. P. Wennerberg et al., "Chromosomal abnormalities involving $11 \mathrm{q} 13$ are associated with poor prognosis in patients with squamous cell carcinoma of the head and neck," Cancer, vol. 76, no. 5, pp. 853-859, 1995.

[59] J. G. Izzo, V. A. Papadimitrakopoulou, X. Q. Li et al., "Dysregulated cyclin D1 expression early in head and neck tumorigenesis in vivo evidence for an association with subsequent gene amplification," Oncogene, vol. 17, no. 18, pp. 2313-2322, 1998.

[60] H. J. Roh, D. M. Shin, J. S. Lee et al., "Visualization of the timing of gene amplification during multistep head and neck tumorigenesis," Cancer Research, vol. 60, no. 22, pp. 6496-6502, 2000.

[61] D. L. Uhlman, G. Adams, D. Knapp, D. M. Aeppli, and G. Niehans, "Immunohistochemical staining for markers of future neoplastic progression in the larynx," Cancer Research, vol. 56, no. 9, pp. 2199-2205, 1996.

[62] V. A. Papadimitrakopoulou, J. Izzo, J. Keck et al., "Cyclin D1 and p16 alterations in advanced premalignant lesions of the upper aerodigestive tract: role in response to chemoprevention and cancer development," Clinical Cancer Research, vol. 7, no. 10, pp. 3127-3134, 2001.

[63] Y. Yamada, R. Pannell, A. Forster, and T. H. Rabbitts, "The oncogenic LIM-only transcription factor Lmo2 regulates angiogenesis but not vasculogenesis in mice," Proceedings of the National Academy of Sciences of the United States of America, vol. 97, no. 1, pp. 320-324, 2000.

[64] N. Yamagata, K. Furuno, M. Sonoda, H. Sugimura, and K. Yamamoto, "Stomach cancer-derived del223V-226L mutation of the STCH gene causes loss of sensitization to TRAIL-mediated apoptosis," Biochemical and Biophysical Research Communications, vol. 376, no. 3, pp. 499-503, 2008. 


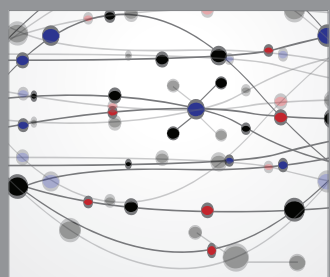

The Scientific World Journal
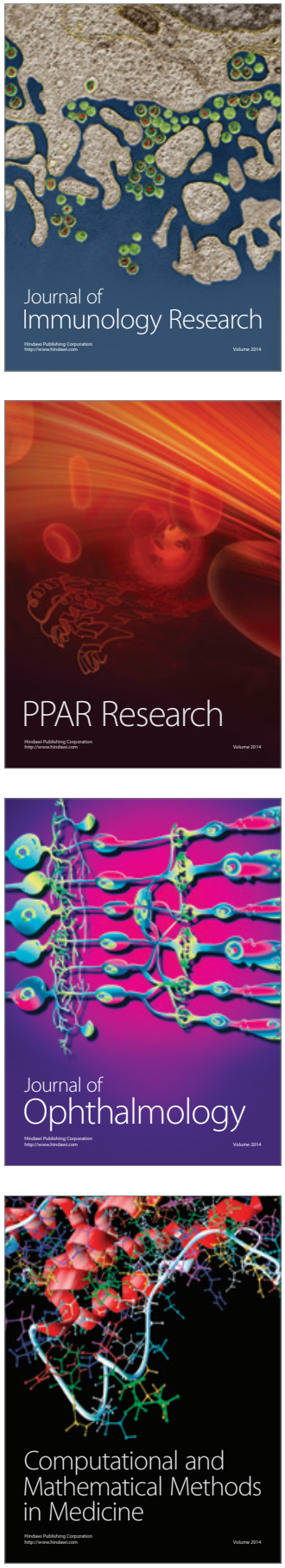

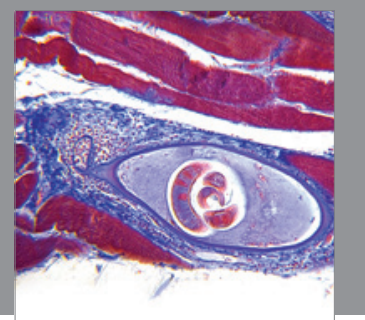

Gastroenterology

Research and Practice
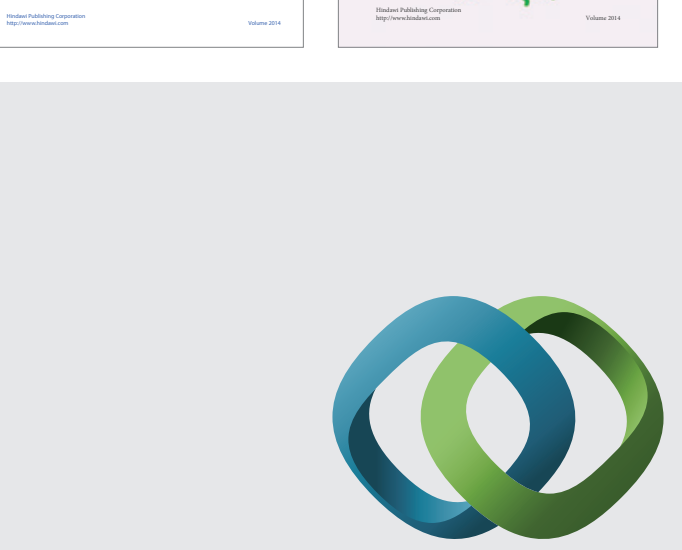

\section{Hindawi}

Submit your manuscripts at

http://www.hindawi.com
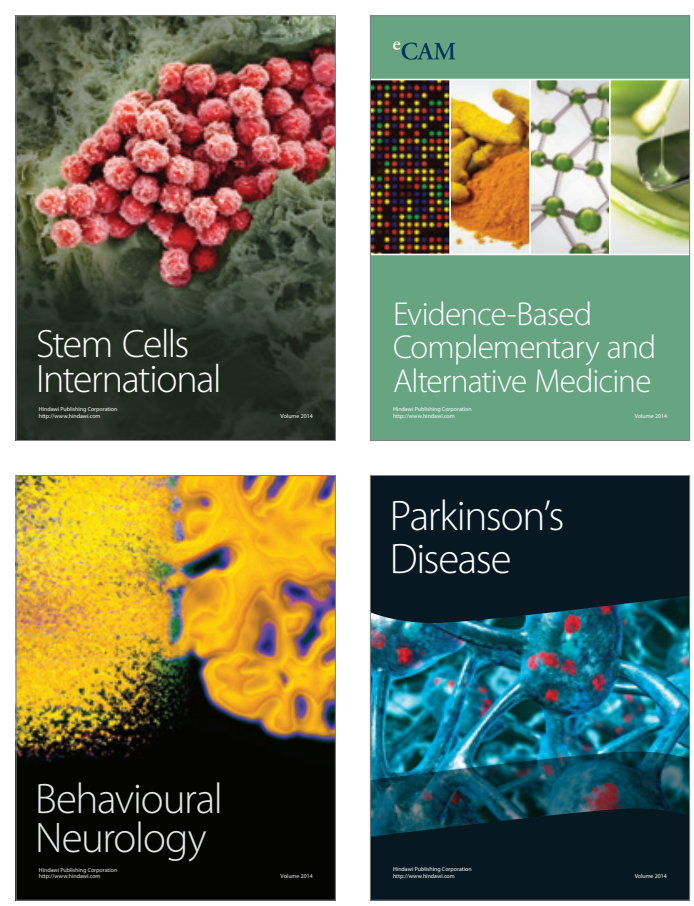

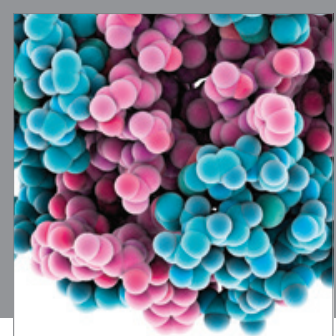

Journal of
Diabetes Research

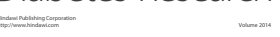

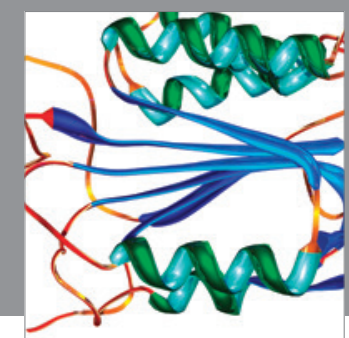

Disease Markers
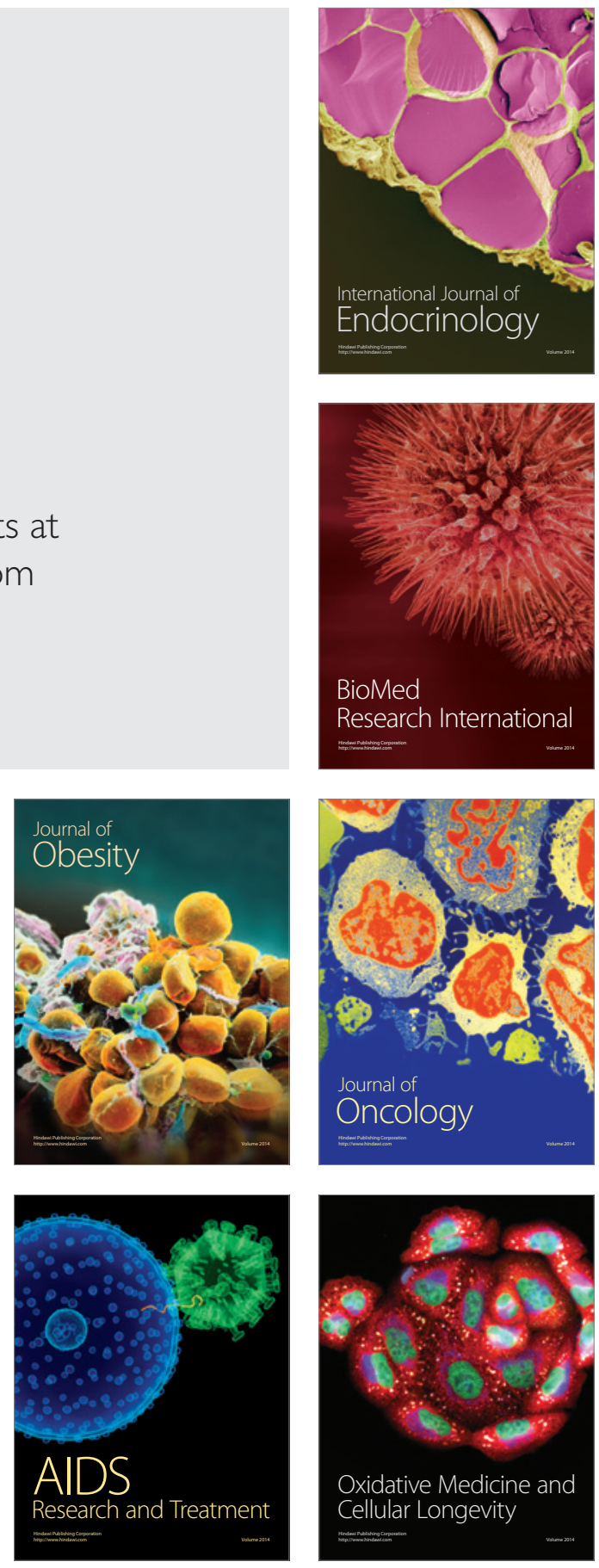